\title{
The Search for Correlation between BiSON SMMF Data and CME Events
}

\author{
William J. Chaplin, Andrew M. Dumbill, Yvonne P. Elsworth, George
} R. Isaak, Clive P. McLeod, Brek A. Miller

School of Physics and Astronomy, University of Birmingham, Edgbaston, Birmingham B15 2TT, UK

\section{Roger New, Balázs Pintér}

School of Science and Mathematics, Sheffield Hallam University, Howard Street, Sheffield S1 $1 \mathrm{WB}, U K$

\begin{abstract}
We present first attempts to compare the Birmingham SolarOscillations Network (BiSON) high precision solar mean magnetic field (SMMF) data of four years with the occurrence of CMEs (coronal mass ejections) as recorded by LASCO on board SOHO. The BiSON magnetic measurement technique is given in Chaplin et al. (2003). Particularly interesting results of recent SMMF high-cadence observations have come from studies of correlation between the SMMF determined by MDI and the occurrence of CMEs (Boberg and Lundstedt 2000 and Boberg et al 2002). Two frequency ranges, centered on 13 and 90 minutes, have been identified as possibly correlating with CME occurrence.

We have used BiSON SMMF data from two sites to investigate CME related SMMF signals to try to confirm the MDI results. To search methodically through our data set we have developed two correlation techniques suited to short (up to 32 minutes) and long (up to 3 hours) period wavelets, respectively. For short periods we analyzed SMMF data in the vicinity of CMEs, and for long periods we compared SMMF results for days with and without recorded CMEs. In neither period range have we yet clearly identified correlations between SMMF power excesses and CME onsets. For the details of the techniques and the results see Chaplin et al. (2004).
\end{abstract}

\section{References}

Boberg F., Lundstedt H., Geophysical Research Letters, 2000, 27, 3141

Boberg F., Lundstedt H., Hoeksema J.T., Scherrer P. H., Liu W., Journal of Geophysical Research, 2002, 107, A10, 1318

Chaplin, W.J., Dumbill A.M., Elsworth Y., Isaak G. R. McLeod C.P., Miller, New R., Pintér B. 2003, MNRAS, 343, 813

Chaplin, W.J., Dumbill A.M., Elsworth Y., Isaak G. R. McLeod C.P., Miller, New R., Pintér B. 2004, Solar Phys., 220, 307 